\title{
Homotopy automorphisms of $R$-module bundles, and the $K$-theory of string topology
}

\author{
Ralph L. Cohen * \\ Department of Mathematics \\ Stanford University \\ Bldg. 380 \\ Stanford, CA 94305, USA
}

\author{
John D.S Jones \\ Mathematics Institute \\ Zeeman Building \\ Warwick University \\ Coventry, CV4 7AL, UK
}

September 9, 2018

\begin{abstract}
Let $R$ be a ring spectrum and $\mathcal{E} \rightarrow X$ an $R$-module bundle of rank $n$. Our main result is to identify the homotopy type of the group-like monoid of homotopy automorphisms of this bundle, $h A u t^{R}(\mathcal{E})$. This will generalize the result regarding $R$-line bundles proven by the authors in 9. The main application is the calculation of the homotopy type of $B G L_{n}(E n d((\mathcal{L}))$ where $\mathcal{L} \rightarrow X$ is any $R$-line bundle, and $\operatorname{End}(\mathcal{L})$ is the ring spectrum of endomorphisms. In the case when such a bundle is the fiberwise suspension spectrum of a principal bundle over a manifold, $G \rightarrow P \rightarrow M$, this leads to a description of the $K$-theory of the string topology spectrum in terms of the mapping space from $M$ to $B G L\left(\Sigma^{\infty}\left(G_{+}\right)\right)$.
\end{abstract}

\section{Contents}

1 Automorphisms of $R$-module bundles

$2 K$-theoretic implications 6

\section{Introduction}

Let $R$ be a ring spectrum. In several places in the recent literature, the notion of an $R$-module bundle $\mathcal{E} \rightarrow X$ of rank $n$ has been defined and described [1, 2], 12. This is a parameterized $R$-module spectrum $\mathcal{E}$ over $X$, where each fiber $E_{x}$ admits an $R$-module equivalence $E_{x} \stackrel{\simeq}{\rightarrow} \vee_{n} R$.

\footnotetext{
${ }^{*}$ The first author was partially supported by a grant from the NSF.
} 
In analogy to vector bundles, it was proved in [12] that equivalence classes of rank $n R$-module bundles over $X$ are in bijective correspondence with the set of homotopy classes, $\left[X, B G L_{n}(R)\right]=$ $\pi_{0}\left(\operatorname{Map}\left(X, B G L_{n}(R)\right)\right.$.

The main theorem in this paper is the identification of the homotopy type of the group-like monoid of homotopy automorphisms, $h A u t^{R}(\mathcal{E})$. This is the space of self equivalences of $\mathcal{E}$ living over the identity of $X$ that preserve the $R$-module structure. A precise definition will be given in the text of the paper.

Theorem 1. Let $R$ be a ring spectrum and $X$ a connected space of the homotopy type of a $C W$ complex. Let $\mathcal{E} \rightarrow X$ be an $R$-module bundle of rank $n$. Then there is an equivalence of group-like monoids,

$$
h A u t^{R}(\mathcal{E}) \simeq \operatorname{SMap}_{\mathcal{E}}\left(X, B G L_{n}(R)\right)
$$

where the subscript in this mapping space refers to the path component of maps that classify $\mathcal{E}$.

The special case of this theorem when $n=1$ was proved by the authors in [9. As discussed there, this result is important in string topology. Namely, given a principal bundle over a manifold $G \rightarrow P \rightarrow M$, if we let

$$
\mathcal{L}=\Sigma_{M}^{\infty}\left(P_{+}\right)
$$

be the fiberwise suspension spectrum of $P$ with a fiberwise disjoint basepoint, then the string topology spectrum of $P, \mathcal{S}(P)=P^{-T M}$ is equivalent, as ring spectra, to the endomorphism ring $\operatorname{End}^{\Sigma^{\infty}\left(G_{+}\right)}\left(\Sigma_{M}^{\infty}\left(P_{+}\right)\right)$. Thus if $R=\Sigma^{\infty}\left(G_{+}\right)$, and $\mathcal{E}=\Sigma_{M}^{\infty}\left(P_{+}\right)$, then the above theorem, in the case $n=1$, describes the homotopy type of the group-like monoid of units, $G L_{1}(\mathcal{S}(P))$.

Theorem 1 in its general setting will have following implication to string topology.

Let $\mathcal{L} \rightarrow X$ be an $R$-line bundle, and let $\oplus_{n} \mathcal{L} \rightarrow X$ be the Whitney-sum of $n$-copies of $\mathcal{L}$. This is an $R$-module bundle of rank $n$.

Corollary 2. There is a homotopy equivalence

$$
B G L_{n}\left(\operatorname{End}_{X}^{R} \mathcal{L}\right) \simeq \operatorname{Map}_{\oplus_{n} \mathcal{L}}\left(X, B G L_{n} R\right) .
$$

As a special case we obtain the following result about the general linear groups of the string topology spectrum.

Corollary 3. If $G \rightarrow P \rightarrow M$ is a principal bundle over a manifold and $\mathcal{L}=\Sigma_{M}^{\infty}\left(P_{+}\right)$, there is a homotopy equivalence

$$
B G L_{n}(\mathcal{S}(P)) \simeq M a p_{\oplus_{n} \mathcal{L}}\left(M, B G L_{n}\left(\Sigma^{\infty}\left(G_{+}\right)\right)\right.
$$

In particular there is an equivalence,

$$
B G L_{n}\left(L M^{-T M}\right) \simeq \operatorname{Map}_{\iota_{n}}\left(M, B G L_{n}\left(\Sigma^{\infty}\left(\Omega M_{+}\right)\right) .\right.
$$


Here $\iota_{n}$ classifies $\oplus_{n} \mathcal{L}$, where $\mathcal{L}=\Sigma_{M}^{\infty}\left(\mathcal{P}_{+}\right)$, and $\mathcal{P} \rightarrow M$ is a universal bundle in the sense that $\mathcal{P}$ is contractible.

Our next main result describes how these results have $K$-theoretic consequences for these ring spectra. First note that the string topology spectrum $\mathcal{S}(P)$ is a nonconnective ring spectrum. Indeed its homology groups are nontrivial through dimension $-n$, where $n$ is the dimension of the manifold $M$. If $\mathcal{S}$ is a ring spectrum, let $K_{\text {conn }}(S)$ denote the algebraic $K$-theory spectrum of its connective cover $K\left(\mathcal{S}_{0}\right)$. This $K$-theory spectrum has zero-space $\Omega^{\infty} K_{\text {conn }}(\mathcal{S})=K_{0}\left(\pi_{0}(\mathcal{S})\right) \times B G L(\mathcal{S})^{+}$, where the superscript + denotes a group completion that will be described in the text of the paper. We will show that Corollary 8 implies the following result about $K$-theory.

Theorem 4. Given an $R$-line bundle $\mathcal{L} \rightarrow M$, there is a homology isomorphism

$$
\alpha: \operatorname{Map}_{\mathcal{L}}\left(M, B G L\left(\Sigma^{\infty}\left(G_{+}\right)\right)\right) \rightarrow \Omega_{0}^{\infty} K_{\text {conn }}(\mathcal{S}(P)) .
$$

The subscript 0 denotes the path component of the basepoint in $\Omega^{\infty} K_{\text {conn }}(\mathcal{S}(P)) . M_{p_{\mathcal{L}}}\left(M, B G L\left(\Sigma^{\infty}\left(G_{+}\right)\right)\right.$ is the homotopy colimit of the mapping spaces $\operatorname{Map}_{\oplus_{n} \mathcal{L}}\left(M, B G L_{n}\left(\Sigma^{\infty}\left(G_{+}\right)\right)\right.$.

This theorem can be viewed as a statement about the group completion of the above mapping spaces. However we point out that this is not the same as the mapping space to the group completion, which would be the zero space of the mapping spectrum $\operatorname{Map}_{0}\left(M, K\left(\Sigma^{\infty}\left(G_{+}\right)\right)\right.$. This spectrum calculates the $K\left(\Sigma^{\infty}\left(G_{+}\right)\right)$-cohomology of $M$. However, as we will show below, we can define a homomorphism of $K$-theory groups,

$$
\gamma: K_{\text {conn }}^{-q}(\mathcal{S}(P)) \rightarrow K\left(\Sigma^{\infty}\left(G_{+}\right)\right)^{-q}(M)
$$

which gives a partial geometric understanding of the $K\left(\Sigma^{\infty}\left(G_{+}\right)\right)$-cohomology theory in terms of the algebraic $K$-theory of the string topology spectrum. The situation when $q=0$ was studied in detail by Lind in [12].

We conclude by observing two important applications of Theorem 4 .

Corollary 5. Let $M$ be a closed manifold. There are homology equivalences

$$
\begin{aligned}
\operatorname{Map}_{\Sigma_{M}^{\infty}\left(\mathcal{P}_{+}\right)}\left(M, B G L\left(\Sigma^{\infty}\left(\Omega M_{+}\right)\right)\right. & \rightarrow \Omega_{0}^{\infty} K_{\text {conn }}\left(L M^{-T M}\right) \\
\operatorname{Map}_{\mathbb{S}}(M, B G L(\mathbb{S})) & \rightarrow \Omega_{0}^{\infty} K_{\text {conn }}(D M)
\end{aligned}
$$

where $\mathbb{S}$ is the sphere spectrum, and $L M^{-T M}$ is the Thom spectrum of the virtual bundle $-T M$ over $M$, pulled back over $L M$ via the map e $: L M \rightarrow M$ that evaluates a loop at the basepoint of the circle. DM denotes the Spanier-Whitehead dual of the manifold $M$, which is an $E_{\infty}$-ring spectrum.

We point out that in these cases, the map $\gamma$ defined above (1) gives homomorphisms

$$
\gamma: K_{\text {conn }}^{-q}\left(L M^{-T M}\right) \rightarrow A(M)^{-q}(M) \quad \text { and } \quad \gamma: K_{\text {conn }}^{-q}(D(M)) \rightarrow A(\text { point })^{-q}(M) .
$$


The algebraic $K$-theory of nonconnective spectra was defined in terms of Waldhausen categories of modules by Blumberg and Mandell in [5. When $X$ is simply connected, they related the Waldhausen category defining $K(D(X))$ to the Waldhausen category defining $A(X)=K\left(\Sigma^{\infty}\left(\Omega X_{+}\right)\right)$. It would be interesting to relate Corollary 5 regarding the $K_{c o n n}$-theory to their results.

This paper is organized as follows. In Section 1 we will prove Theorem 1 and derive Corollaries 7 and 8 . In Section 2 we describe the $K$-theoretic implications of Thoerem 1, and in particular we prove Theorem 4 .

\section{Automorphisms of $R$-module bundles}

Let $R$ be a ring spectrum. Let $\mathcal{E} \rightarrow X$ be an $R$-module bundle of rank $n$, in the sense of Lind [12]. This is a parameterized spectrum over $X$ in the sense of May and Sigurdsson [13, where for each $x \in X$, the fiber $\mathcal{E}_{x}$ is an $R$-module spectrum of rank $n$. We denote the category of such bundles by $R-\bmod _{n}(X)$. Again, this category was defined in [12]. It was shown there that equivalence classes of such bundles are classified by homotopy classes of maps $X \rightarrow B G L_{n}(R)$. Fix a particular map $\gamma_{\mathcal{E}}: X \rightarrow B G L_{n}(R)$ classifying $\mathcal{E}$. This choice defines a basepoint in the mapping space $\gamma_{\mathcal{E}} \in \operatorname{Map}_{\mathcal{E}}\left(X, B G L_{n}(R)\right)$. The endomorphisms of $\mathcal{E}$ in $R-\bmod _{n}(X)$ is a parameterized spectrum which we denote by $\operatorname{End} d_{M}^{R}(\mathcal{E}) \rightarrow X$. For every $x \in X$ this defines a fiber spectrum $\operatorname{End}_{M}^{R}(\mathcal{E})_{x}$ which is equivalent to the ring of endomorphisms $\operatorname{End}^{R}\left(\vee_{n} R\right)$. $\operatorname{End}_{M}^{R}(\mathcal{E})$ is a parameterized ring spectrum under composition. By taking a fibrant replacement if necessary, we can take sections to produce an ordinary spectrum

$$
\operatorname{End}^{R}(\mathcal{E})=\Gamma_{M}\left(\operatorname{End}_{M}^{R}(\mathcal{E})\right)
$$

The parameterized ring structure on $\operatorname{End}_{M}^{R}(\mathcal{E})$ defines a ring spectrum structure on $\operatorname{End}^{R}(\mathcal{E})$.

Definition 1. We define the group-like monoid $h A u t^{R}(\mathcal{E})$ to be the units of the ring spectrum of endomorphisms,

$$
h A u t^{R}(\mathcal{E})=G L_{1}\left(E n d^{R}(\mathcal{E})\right)
$$

We are now ready to prove Theorem 1

Proof. Consider the fiber bundle of infinite loop spaces given by taking the zero spaces of the fibrant model of $\operatorname{End}_{M}^{R}(\mathcal{E})$ :

$$
\Omega^{\infty}\left(\operatorname{End}^{R}\left(\vee_{n} R\right)\right) \rightarrow \Omega_{M}^{\infty} \operatorname{End}_{M}^{R}(\mathcal{E}) \rightarrow M
$$

By restricting to path components of those $R$-module endomorphisms that consist of equivalences, we get a subbundle, which we will call $\mathcal{G} L_{n}(\mathcal{E})$ :

$$
G L_{n}(R) \rightarrow \mathcal{G} L_{n}(\mathcal{E}) \rightarrow M .
$$


Notice that $h A u t^{R}(\mathcal{E})$ can be described as the space of sections of this bundle, $h A u t^{R}(\mathcal{E})=$ $\Gamma_{M}\left(\mathcal{G} L_{n}(\mathcal{E})\right)$. We now observe that the fiber homotopy type of the bundle $\mathcal{G} L_{n}(\mathcal{E}) \rightarrow M$ has another description. The homotopy class of map $\gamma_{\mathcal{E}}: M \rightarrow B G L_{n}(R)$ that classifies the $R$-module bundle $\mathcal{E}$, also classifies a principal $G L_{n}(R)$-bundle over $M$ :

$$
G L_{n}(R) \rightarrow P_{\mathcal{E}} \rightarrow M
$$

Here we are replacing the group-like topological monoid $G L_{n}(R)$ by a topological group, which by abuse of notation, we continue to refer to as $G L_{n}(R)$. As was shown in [12, the relationship between $P_{\mathcal{E}}$ and the parameterized spectrum $\mathcal{E}$ is that there is an equivalence,

$$
\Omega_{M}^{\infty} \mathcal{E} \simeq P_{\mathcal{E}} \times_{G L_{n}(R)} \Omega^{\infty}\left(\vee_{n} R\right),
$$

where we are continuing the abuse of notation to allow $\Omega^{\infty}\left(\vee_{n} R\right)$ to refer to a weakly homotopy equivalent infinite loop space that carries an action of the group $G L_{n} R$. (See [13] and [12] for details.)

Now consider the corresponding adjoint bundle, $G L_{n}(R) \rightarrow P_{\mathcal{E}}^{A d} \rightarrow X$. In this notation $P_{\mathcal{E}}^{A d}$ is the homotopy orbit space $P_{\mathcal{E}}^{A d}=P_{\mathcal{E}} \times_{G L_{n}(R)} G L_{n}(R)$ where $G L_{n}(R)$ is acting on itself by the adjoint action (conjugation). A standard observation about adjoint bundles implies that $P_{\mathcal{E}}^{A d}$ is the bundle whose fiber over $x \in M$ is the space of $G L_{n}(R)$-equivariant automorphisms of the fiber of $P_{\mathcal{E}}$ at $x$. The section space $\Gamma_{M}\left(P_{\mathcal{E}}^{A d}\right)$ is therefore the group of equivariant automorphisms of $P_{\mathcal{E}}$ living over the identity on $M$. This is known as the gauge group, $\mathcal{G}\left(P_{\mathcal{E}}\right)$.

From this viewpoint it becomes clear that there is a map of fibrations $P_{\mathcal{E}}^{A d} \rightarrow \mathcal{G} L_{n}(\mathcal{E})$ over $M$ (after taking appropriate fibrant and cofibrant replacements), which is an equivalence on the fibers. Therefore there is an equivalence of their spaces of sections, as group-like $A_{\infty}$-spaces.

$$
\phi: \mathcal{G}\left(P_{\mathcal{E}}\right)=\Gamma_{M}\left(P_{\mathcal{E}}^{A d}\right) \simeq \Gamma_{M}\left(\mathcal{G} L_{1}(\mathcal{E})\right)=h A u t_{X}^{R}(\mathcal{E}) .
$$

Now a well known theorem of Atiyah and Bott [4 says that the classifying space of the gauge group $\mathcal{G}(P)$ of a principal bundle $G \rightarrow P \rightarrow M$ is equivalent to the mapping space, $B \mathcal{G}(P) \simeq$ $\operatorname{Map}_{P}(X, B G)$. Theorem 1 now follows by applying this Atiyah-Bott equivalence to the principal bundle $G L_{n}(R) \rightarrow P_{\mathcal{E}} \rightarrow X$.

We now consider an application of Theorem 1 to an important special case. Let $\mathcal{L} \rightarrow X$ be an $R$-line bundle, and let $\oplus_{n} \mathcal{L} \rightarrow X$ be the Whitney-sum of $n$-copies of $\mathcal{L}$. This is an $R$-module bundle of rank $n$, which is defined to be the pullback under the diagonal map $\Delta^{n}: X \rightarrow X^{n}$ of the exterior $n$-fold product $\mathcal{L}^{n} \rightarrow X^{n}$. Consider the endomorphism spectrum $\operatorname{End}^{R}(\mathcal{L})$. As noted above, this is a ring spectrum. We first need the following observation:

Lemma 6. There is an equivalence of group-like monoids

$$
\lambda: h A u t^{R}\left(\oplus_{n} \mathcal{L}\right) \simeq G L_{n}\left(\operatorname{End}^{R}(\mathcal{L})\right)
$$


Proof. Given a ring spectum $S$, recall that $G L_{n}(S)$ is defined to be the group-like monoid of units in the endomorphism ring,

$$
G L_{n}(S)=G L_{1}\left(E n d^{S}\left(\vee_{n} S\right)\right)
$$

Notice that there is a natural equivalence $E n d^{S}\left(\vee_{n} S\right)=\prod_{n}\left(\vee_{n} S\right)$. Also notice that $\vee_{n} E n d^{R}(\mathcal{L}) \simeq$ $\operatorname{Mor}^{R}\left(\mathcal{L}, \oplus_{n} \mathcal{L}\right)$, where $\operatorname{Mor}^{R}$ refers to the $R$-module morphisms in the category of parameterized spectra over $X$. Thus when $S=E n d^{R}(\mathcal{L})$, we have a natural equivalence

$$
\operatorname{End}^{S}\left(\vee_{n} S\right) \simeq \prod_{n}\left(\operatorname{Mor}^{R}\left(\mathcal{L}, \oplus_{n} \mathcal{L}\right)=\operatorname{End}^{R}\left(\oplus_{n} \mathcal{L}\right)\right.
$$

Furthermore this equivalence clearly preserves the ring structure. It therefore induces an equivalence of their group-like monoids of units,

$$
G L_{1}\left(\operatorname{End}^{S}\left(\vee_{n} S\right)\right) \simeq G L_{1}\left(\operatorname{End}^{R}\left(\oplus_{n} \mathcal{L}\right) .\right.
$$

The left side is by definition $G L_{n}\left(E n d^{R}(\mathcal{L})\right)$, and the right side is by definition $h A u t^{R}\left(\oplus_{n} \mathcal{L}\right)$.

We now notice that Theorem 1 and Lemma 6 together imply the following.

Corollary 7. There is a homotopy equivalence

$$
B G L_{n}\left(E n d_{X}^{R} \mathcal{L}\right) \simeq \operatorname{Map}_{\oplus_{n} \mathcal{L}}\left(X, B G L_{n} R\right)
$$

When $G \rightarrow P \rightarrow M$ is a principal bundle over a manifold and $\mathcal{L}=\Sigma_{M}^{\infty}\left(P_{+}\right)$, we obtain the following result about the general linear groups of the string topology spectrum,

Corollary 8. There is a homotopy equivalence

$$
\beta: B G L_{n}(\mathcal{S}(P)) \stackrel{\simeq}{\longrightarrow} \operatorname{Map}_{\oplus_{n} \mathcal{L}}\left(M, B G L_{n}\left(\Sigma^{\infty}\left(G_{+}\right)\right)\right.
$$

where $\mathcal{L}$ is the line bundle $\Sigma^{\infty}\left(G_{+}\right) \rightarrow \Sigma_{M}^{\infty}\left(P_{+}\right) \rightarrow M$. In particular there is an equivalence

$$
B G L_{n}\left(L M^{-T M}\right) \simeq \operatorname{Map}_{\iota_{n}}\left(M, B G L_{n}\left(\Sigma^{\infty}\left(\Omega M_{+}\right)\right) .\right.
$$

\section{$2 \quad K$-theoretic implications}

The goal of this section is to use Corollary 8 to prove Theorem 4 as stated in the introduction. We will then use it to derive descriptions of the $K$-theory of the connective covers of the Spanier-Whitehead dual $D(M)=\operatorname{Map}\left(\Sigma^{\infty}\left(M_{+}\right), \mathbb{S}\right)$ and of the string topology ring spectrum $L M^{-T M}$.

To do this we need to understand the equivalence given in Corollary 7 more carefully, so that we can deduce $K$-theoretic consequences. As in that corollary, let $\mathcal{L} \rightarrow M$ be an $R$-line bundle and let $P_{\mathcal{L}} \rightarrow M$ denote the principal $G L_{1} R$-bundle defined by the classifying map of $\mathcal{L}, \gamma_{\mathcal{L}}: M \rightarrow$ $B G L_{1}(R)$. Let $\oplus_{n} P_{\mathcal{L}}$ denote the principal bundle classified by

$$
M \stackrel{\Delta}{\rightarrow} \prod_{n} M \stackrel{\gamma_{\mathcal{L}}^{n}}{\longrightarrow} \prod_{n} B G L_{1}(R) \stackrel{\mu}{\rightarrow} B G L_{n}(R) .
$$


Here $\mu$ is the usual block addition. This is the principal $G L_{n}(R)$-bundle associated to the $n$-fold Whitney sum $\oplus_{n} \mathcal{L}$.

Combining equivalence (2) with Lemma 6 defines an equivalence of group-like $A_{\infty}$-spaces,

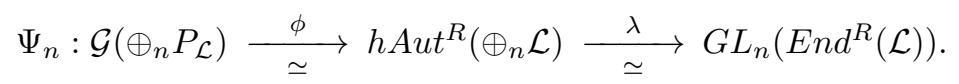

Notice that these equivalences respect the standard inclusions of wreath products, that makes the following diagrams commute:

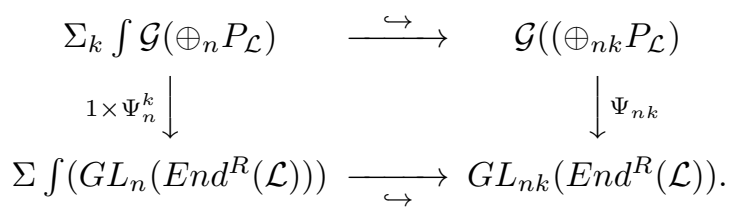

Using the Barratt-Eccles $E_{\infty}$-operad, we can conclude the following:

Proposition 9. The disjoint unions $\coprod_{n \geq 0} B \mathcal{G}\left(\oplus_{n} P_{\mathcal{L}}\right)$ and $\coprod_{n \geq 0} B G L_{n}\left(\operatorname{End}^{R}(\mathcal{L})\right)$ have $E_{\infty}$-algebra structures, and the equivalence

$$
\sqcup B \Psi_{n}: \coprod_{n \geq 0} B \mathcal{G}\left(\oplus_{n} P_{\mathcal{L}}\right) \rightarrow \coprod_{n \geq 0} B G L_{n}\left(\operatorname{End}^{R}(\mathcal{L})\right)
$$

is an equivalence of $E_{\infty}$-spaces.

We are now ready to deduce $K$-theoretic information from the above analysis.

If $X$ is an $E_{\infty}$ space, let $X^{+}$denote the corresponding infinite loop space given by its group completion. In other words,

$$
X^{+}=\Omega B(X)
$$

Given a ring spectrum $S$, let $S_{0}$ denote its connective cover. We denote by $K_{\text {conn }}(S)$ the algebraic $K$-theory spectrum $K\left(S_{0}\right)$. A connected component of the zero space of this spectrum is given by $\Omega_{0}^{\infty} K_{\text {conn }}(S)=\left(\amalg_{n} B G L_{n}(S)\right)_{0}^{+}$, the component of the basepoint in the group completion. We note that the usual algebraic $K$-theory spectrum $K(S)$ is defined as the $K$-theory of the Waldhausencategory of perfect modules over $S[5]$. When $S$ is not connective, these spectra are not necessarily equivalent, where they are for connective ring spectra.

We can now conclude the following.

Proposition 10. If $R$ is a ring spectrum and $R \rightarrow \mathcal{L} \rightarrow M$ is an $R$-line bundle over a closed manifold $M$, then each path component of the zero space of the $K$-theory spectrum of the endomorphism ring has the following homotopy type:

$$
\Omega_{0}^{\infty}\left(K_{\text {conn }}\left(\operatorname{End}^{R}(\mathcal{L})\right)\right) \simeq\left(\coprod_{n \geq 0} B G L_{n}\left(E n d^{R}(\mathcal{L})\right)\right)_{0}^{+} \simeq\left(\coprod_{n \geq 0} B \mathcal{G}\left(\oplus_{n} P_{\mathcal{L}}\right)\right)_{0}^{+} .
$$


We now proceed with the proof of Theorem 4 as stated in the introduction.

Proof. As is usual, let $B G L(\operatorname{End}(\mathcal{L}))$ denote the homotopy colimit of the inclusion maps $B G L_{n}(E n d(\mathcal{L})) \hookrightarrow$ $B G L_{n+1}(\operatorname{End}(\mathcal{L}))$ induced by the usual inclusions of the groups $G L_{n}(\operatorname{End}(\mathcal{L})) \hookrightarrow G L_{n+1}(\operatorname{End}(\mathcal{L}))$. Then by the group-completion theorem of McDuff-Segal [14, there is a homology equivalence,

$$
\alpha: \mathbb{Z} \times B G L(\operatorname{End}(\mathcal{L})) \rightarrow\left(\coprod_{n \geq 0} B G L_{n}\left(\operatorname{End}^{R}(\mathcal{L})\right)\right)^{+} \simeq \mathbb{Z} \times \Omega_{0}^{\infty}\left(K_{\text {conn }}\left(\operatorname{End}^{R}(\mathcal{L})\right)\right) .
$$

Furthermore, by Proposition 10 there is an equivalence

$$
\mathbb{Z} \times \operatorname{hocolim}_{n} B \mathcal{G}\left(\oplus_{n} P_{\mathcal{L}}\right) \simeq \mathbb{Z} \times B G L(\operatorname{End}(\mathcal{L}))
$$

The maps in the colimit are induced by the inclusions of gauge groups, $j_{n}: \mathcal{G}\left(\oplus_{n} P_{\mathcal{L}}\right) \hookrightarrow \mathcal{G}\left(\oplus_{n+1} P_{\mathcal{L}}\right)$ given by thinking of an automorphism of $\oplus_{n} P$ as an automorphism of $\oplus_{n+1} P$ by taking the direct sum with the identity map on the last factor.

Now for any principal bundle $G \rightarrow P \rightarrow M$, consider the Atiyah-Bott equivalence [4] mentioned in the proof of Theorem 1

$$
\beta: B \mathcal{G}(P) \simeq \operatorname{Map}_{P}(M, B G) .
$$

This equivalence is given by the observation that the equivariant mapping space $\operatorname{Map}^{G}(P, E G)$ is a contractible space with a free action of the gauge group $\mathcal{G}(P)=A u t_{M}^{G}(P)$ given by precomposition. The orbit space of this action is then $\operatorname{Map}_{P}(M, B G)$. The examples relevant here are the principal bundles $G L_{n}(R) \rightarrow \oplus_{n} P_{\mathcal{L}} \rightarrow M$. In this case we have an equivalence

$$
\beta_{n}: B \mathcal{G}\left(\oplus_{n} P_{\mathcal{L}}\right) \stackrel{\simeq}{\rightarrow} \operatorname{Map}_{\oplus_{n} \mathcal{L}}\left(M, B G L_{n} R\right)
$$

With respect to these equivalences, the inclusions $j_{n}: B \mathcal{G}\left(\oplus_{n} P_{\mathcal{L}}\right) \rightarrow B \mathcal{G}\left(\oplus_{n+1} P_{\mathcal{L}}\right)$ are given up to homotopy by maps

$$
q_{n}: \operatorname{Map}_{\oplus_{n} \mathcal{L}}\left(M, B G L_{n} R\right) \rightarrow \operatorname{Map}_{\oplus_{n+1} \mathcal{L}}\left(M, B G L_{n+1} R\right)
$$

defined by sending $\phi: M \rightarrow B G L_{n}(R)$ to the composition

$$
q_{n}(\phi): M \stackrel{\Delta}{\rightarrow} M \times M \stackrel{\phi \times \gamma_{\mathcal{L}}}{\longrightarrow} B G L_{n}(R) \times B G L_{1}(R) \stackrel{\mu}{\rightarrow} B G L_{n+1}(R)
$$

where $\gamma_{\mathcal{L}}$ is the fixed basepoint in $\operatorname{Map}_{\mathcal{L}}\left(M, B G L_{1}(R)\right)$, and $\mu$ is the usual block pairing.

If we denote the homotopy colimit of these maps hocolim ${ }_{n} M a p_{\oplus_{n}} \mathcal{L}\left(M, B G L_{n} R\right)$ by $M_{a p_{\mathcal{L}}}(M, B G L(R))$, we then have a homology equivalence

$\mathbb{Z} \times \operatorname{Map}_{\mathcal{L}}(M, B G L(R)) \stackrel{\simeq}{\rightarrow} \mathbb{Z} \times \operatorname{hocolim}_{n} B \mathcal{G}\left(\oplus_{n} P_{\mathcal{L}}\right) \simeq \mathbb{Z} \times B G L(\operatorname{End}(\mathcal{L})) \rightarrow \mathbb{Z} \times \Omega_{0}^{\infty} K_{\text {conn }}(\operatorname{End}(\mathcal{L}))$, which is the statement of Theorem 4 
We now proceed to prove Corollary 5 describing $K_{\text {conn }}$ of both the string topology spectrum, and of the Spanier-Whitehead dual of a manifold. In the case of the string topology spectrum $\mathcal{S}(M)=L M^{-T M}$, this is the string topology of the universal principal bundle. That is, a principal bundle over $M$ fiber homotopy equivalent to the path-loop fibration $\Omega M \rightarrow \mathcal{P}_{M} \rightarrow M$. In the case of the Spanier-Whitehead dual, $D M$, one sees that if one considers the trivial principal bundle, when $G=\{i d\}$, then the string topology spectrum of this bundle is simply the dual, $D M=\operatorname{Map}(M, \mathbb{S})$. Both of these examples are analyzed in more detail in $[9$. Corollary 5 follows by applying Theorem 4 to these special cases.

\section{References}

[1] M. Ando, A. J. Blumberg, D. J. Gepner, M. J. Hopkins, and C. Rezk. Units of ring spectra and Thom spectra preprint, arXiv:0810.4535

[2] M. Ando, A. J. Blumberg, D. Gepner, M. J. Hopkins, and C. Rezk, An $\infty$-categorical approach to $R$-line bundles, $R$-module Thom spectra, and twisted $R$-homology, preprint (2012).

[3] M.F. Atiyah, Thom complexes, Proc. London Math. Soc. (3) , no. 11 (1961), 291-310.

[4] M. Atiyah, and R. Bott The Yang-Mills equations over Riemann surfaces, Phil. Trans. R. Soc. Lond. A 308, 523-615 (1982)

[5] A. Blumberg and M. Mandell, Derived Koszul Duality and Involutions in the Algebraic K-Theory of Spaces, J. Topology 4 (2011), no. 2, 327-342, preprint: arXiv:0912.1670

[6] G. Carlsson and R.L. Cohen The free loop space and the cyclic groups, Commentari Math. Helvetici. 62 (1987), 423 - 449.

[7] M. Chas and D. Sullivan, String Topology. preprint: math.GT/9911159.

[8] R.L. Cohen and J.D.S. Jones, A homotopy theoretic realization of string topology, Math. Annalen, vol. 324, 773-798 (2002). preprint: math.GT/0107187

[9] R.L. Cohen and J.D.S. Jones, Gauge theory and string topology, preprint: http://arxiv.org/abs/1304.0613

[10] R.L. Cohen and J.R. Klein, Umkehr maps, Homology, Homotopy, and Applications, vol. 11 (1), (2009), 17-33. preprint: arXiv:0711.0540

[11] K. Gruher and P. Salvatore, Generalized string topology operations Proc. Lond. Math. Soc. (3) 96 (2008), 78106.

[12] J.A. Lind, Bundles of spectra and Algebraic K-theory, preprint arXiv:1304567 
[13] J. P. May and J. Sigurdsson, Parametrized homotopy theory. Mathematical Surveys and Monographs, vol. 132, Amer. Math. Soc., 2006 Mathematical Surveys and Monographs, vol. 132, Amer. Math. Soc., 2006

[14] D. McDuff, G.B. Segal, Homology fibrations and the "group completion" theorem, Invent. Math., 31, (1976), $279-284$. 